\title{
Magnetic resonance tomography of the knee joint
}

\author{
Stefan Puig ${ }^{1} \cdot$ Yojena Chittazhathu Kurian Kuruvilla ${ }^{1} \cdot$ Lukas Ebner $^{1} \cdot$ Gottfried Endel $^{2}$
}

Received: 12 February 2015 /Revised: 30 April 2015 /Accepted: 21 May 2015 /Published online: 1 July 2015

(C) ISS 2015

\begin{abstract}
Objectives To compare the diagnostic performance of magnetic resonance imaging (MRI) in terms of sensitivity and specificity using a field strength of $<1.0 \mathrm{~T}(\mathrm{~T})$ versus $\geq 1.5 \mathrm{~T}$ for diagnosing or ruling out knee injuries or knee pathologies. Methods The systematic literature research revealed more than 10,000 references, of which 1598 abstracts were reviewed and 87 full-text articles were retrieved. The further selection process resulted in the inclusion of four systematic reviews and six primary studies.

Results No differences could be identified in the diagnostic performance of low- versus high-field MRI for the detection or exclusion of meniscal or cruciate ligament tears. Regarding the detection or grading of cartilage defects and osteoarthritis of the knee, the existing evidence suggests that high-field MRI is tolerably specific but not very sensitive, while there is literally no evidence for low-field MRI because only a few studies with small sample sizes and equivocal findings have been performed.

Conclusions We can recommend the use of low-field strength MRI systems in suspected meniscal or cruciate ligament injuries. This does, however, not apply to the diagnosis and
\end{abstract}

Electronic supplementary material The online version of this article (doi:10.1007/s00256-015-2178-5) contains supplementary material, which is available to authorized users.

Stefan Puig

puig1@mac.com

1 Department of Interventional, Pediatric and Diagnostic Radiology Inselspital, University Hospital, University of Berne, Freiburgstr. 10, CH-3010 Berne, Switzerland

2 Main Association of Austrian Social Insurance Institutions, Kundmanngasse 21, A-1030 Vienna, Austria grading of knee cartilage defects and osteoarthritis because of insufficient evidence.

Keywords Low-field magnetic resonance imaging $\cdot$ Meniscal tears $\cdot$ Cruciate ligament tears $\cdot$ Knee cartilage defects $\cdot$ Knee osteoarthritis

\section{Introduction}

In patients who present with knee abnormalities, the first step of diagnosis is usually reviewing the clinical history and performing a physical examination involving various manipulative tests. For patients in which the diagnosis is uncertain or the symptoms persist, physicians must use other diagnostic modalities; in the past, this was usually diagnostic arthroscopy [1]. However, due to its invasive nature, orthopaedic surgeons have increasingly turned to magnetic resonance imaging (MRI), a proven reliable and safe modality that offers a combined evaluation of bones, ligaments, and soft tissue $[2,3]$.

MRI is commonly performed with whole-body high-field scanners using a field strength of $>1.0 \mathrm{~T}$. For specific clinical questions in pathologies of the upper and lower extremities, so-called low- (or medium-) field-strength MR scanners, dedicated to the study of extremities, can be used. Open low-field whole-body scanners are less common. Low-field systems have several advantages and disadvantages [2-6], such as lower costs, but have a lower signal-to-noise ratio leading to lower image quality. These characteristics not only lead to divergences amongst radiologists, but also to recurrent discussions about reimbursement. Thus, the purpose of our study was to compare the diagnostic performance in terms of sensitivity and specificity of MRI using a field strength of $<1.0 \mathrm{~T}$ versus $\geq 1.5 \mathrm{~T}$ for diagnosing or ruling out knee injuries or knee pathologies. 


\section{Materials and methods}

A preliminary review of the literature had revealed that a considerable number of systematic reviews on the topic, published between 2003 and 2012, were already available. Additionally, it was observed that diagnostic accuracy studies using low-field MRI systems were published mainly in the 1990s. We decided to rely primarily on the available systematic reviews to answer our research question provided that they met our inclusion criteria. In case those systematic reviews did not fully meet our needs, additional primary studies were to be considered. Otherwise, we used the recommended methods for systematic reviews of diagnostic accuracy studies [7, 8] and formulated the inclusion and exclusion criteria displayed in Table 1.

For the systematic literature search in the databases Medline, Cochrane Database of Systematic Reviews, and Scopus, combinations of the following search terms were used: magnetic resonance imaging, MR imaging, MRI, MRT, knee, meniscus, cruciate ligament, cartilage, chondral, and arthroscopy. The search was limited to papers published between 1990 and 2013 and not restricted to specific languages. Additionally, we screened the reference lists of the original articles for work that was not found with the described literature search.

All articles that could not be excluded on the basis of the title or abstract of the article were retrieved in full text. One author decided whether the selected studies met the inclusion and exclusion criteria for this review, while two other authors checked whether the selection process was correct.

The literature search revealed 11,987 references, of which 2347 were duplicates and 8042 could be excluded based on the title. Of the remaining 1598 articles, the abstracts were reviewed. Out of these, 87 full-text articles were retrieved. Primary studies that were retrieved in full text but were included in one of the presented systematic reviews were later excluded. This resulted in the final inclusion of four systematic reviews (of totally 94 diagnostic primary studies) and six primary studies. The flowchart of study selection, retrieval, and inclusion is displayed in Fig. 1.

Data were abstracted by the first author (SP) and checked for accuracy and completeness independently by the two coauthors (YCKK, LE). Any disagreements were resolved through discussion. For calculation of pooled sensitivity and specificity values, the absolute numbers of true-positive, falsepositive, true-negative, and false-negative cases per study and type of injury or pathology were considered.

\section{Results}

\section{Quality assessment}

We found four systematic reviews referring to the diagnostic performance of MRI in knee pathologies that met both the inclusion and exclusion criteria. One was published in 2003, one in 2011, and two in 2012. Two included both low- and high-field MRT studies [2,9], and the other two $[10,11]$ had used a minimum field strength of $1.5 \mathrm{~T}$ as an inclusion criteria.

Oei et al. [2] displayed a meta-analysis of 29 articles in their systematic review study to determine the diagnostic performance of all-strength MRI of the menisci and cruciate ligaments and to assess the effect of study design characteristics and magnetic field strength on diagnostic performance. In this work, the eligibility criteria for including articles were clearly specified, data extraction had been performed by two authors independently, publication bias was addressed and determined to be very unlikely because of almost perfectly shaped funnel plots, reasons for excluding full text articles were given, and a check for possibility of verification bias was performed for each article.

Smith et al. [9] evaluated the diagnostic accuracy of anterior cruciate ligament $(A C L)$ rupture using all-strength MRI by performing a pooled meta-analysis. They included 53 articles of which 16 had already been referred to by Oei et al. [2]. The same quality assurance tasks as described in the previous paragraph for the paper of Oei et al. [2] had been performed by these authors; additionally, they had used the QUADAS checklist [12] to assess each study's methodological quality.

Quatman et al. [11] aimed to reveal the clinical utility and diagnostic performance of MRI for the identification of knee osteoarthritis. They found 27 studies of varying quality that met their inclusion criteria including a minimum field strength

Table 1 Criteria for inclusion and exclusion of systematic reviews and primary studies

\begin{tabular}{|c|c|}
\hline \multirow{5}{*}{$\begin{array}{l}\text { Inclusion } \\
\text { criteria }\end{array}$} & Article published in English, German, Spanish, Turkish, French, or Italian language \\
\hline & Depicting lesions of the collateral ligaments, retinacula, or cartilage of the human knee \\
\hline & Magnetic field strength reported \\
\hline & Findings at arthroscopy or surgery or histological workup used as a reference standard \\
\hline & Sensitivity and specificity of MRI and/or rates of true-positive, true negative, false-positive, or false-negative results reported \\
\hline \multirow{2}{*}{$\begin{array}{l}\text { Exclusion } \\
\text { criteria }\end{array}$} & MRI used for postoperative evaluation \\
\hline & $\begin{array}{l}\text { Only the diagnostic value of specific features or indirect signs of knee pathologies at MRI, such as the empty notch sign, anterior tibial } \\
\text { subluxation, or bone bruise, assessed }\end{array}$ \\
\hline
\end{tabular}


Fig. 1 PRISMA flowchart of study selection, retrieval, and inclusion

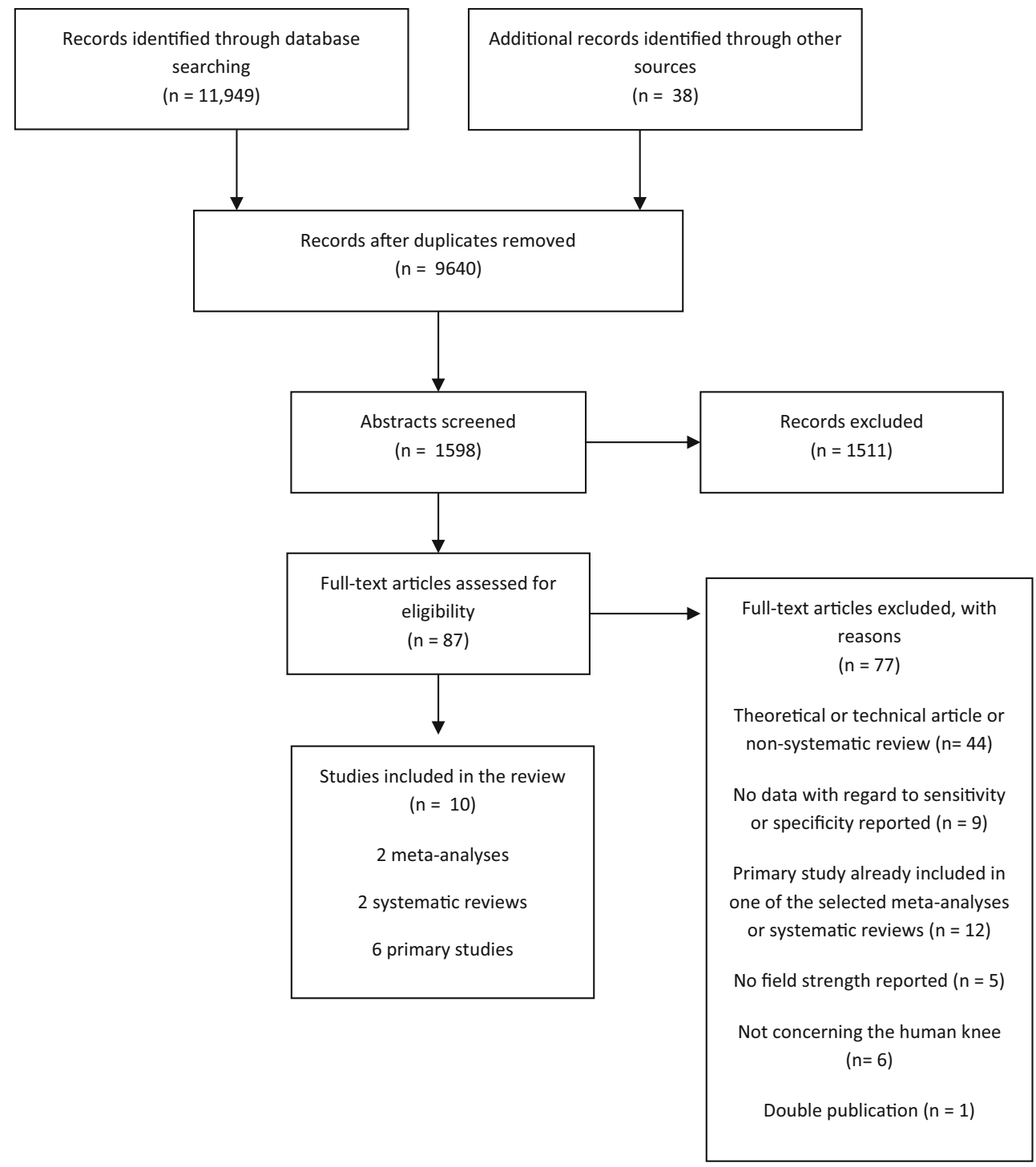

of 1.5 T. The diagnostic performance of MRI demonstrated a wide range, and there were large inconsistencies between imaging techniques, specifically in the heterogeneity of MRI sequences.

Harris et al. [10] presented a systematic review on the sensitivity of MRI for detection of patellofemoral articular cartilage defects. Inclusion criteria were that patients underwent both MRI and arthroscopy and that the minimum field strength was $1.5 \mathrm{~T}$. Thirteen studies were considered in this review, but significant heterogeneity across studies precluded meta-analysis. The quality of the included studies was rather diverse.

The available meta-analyses and systematic reviews did not include low-field MRI studies for the detection of cartilage defects and osteoarthritis of the knee. Therefore, relevant primary studies were assessed. Actually, only a handful of studies that used a reference standard to determine the sensitivity and specificity of low-field MRI was performed or could be identified, respectively. They are displayed in Table 2.
Furthermore, these studies were published between 1995 and 2003, at a time when quality standards for the performance of diagnostic accuracy studies were not yet widely spread. Nevertheless, we tried to describe the methodological strengths and weaknesses of the individual studies considering the questions contained in the QUADAS checklist [12] and summarised those in the "Strengths and weaknesses of the study".

\section{Diagnostic performance}

Data were extracted from two high-quality meta-analyses dealing with the diagnostic accuracy of MRI in knee injuries related to the menisci and the cruciate ligaments [2,9]. Table 3 details the number and content of included articles, publication period of included articles, inclusion criteria, and results with regard to differences in diagnostic performance between low- and high-field MRI . 
Table 2 Methodological strengths and weaknesses of identified studies evaluating low-field MR imaging of knee cartilage defects [13-18]

\begin{tabular}{|c|c|c|c|}
\hline $\begin{array}{l}\text { First author (year } \\
\text { of publication) }\end{array}$ & $\begin{array}{l}\text { Specialisation of } \\
\text { authors }\end{array}$ & Strengths of study & Weaknesses of study \\
\hline Ahn (1998) & $\begin{array}{l}\text { Radiology, orthopaedic } \\
\text { surgery, pathology }\end{array}$ & $\begin{array}{l}\text { Macroscopic findings used as reference; } \\
\text { grading of cartilage lesions considered; } \\
\text { relevance of MRI sequences considered }\end{array}$ & $\begin{array}{l}\text { Only } 10 \text { patellae from human cadavers } \\
\text { evaluated; all cadavers from patients } \\
\text { >78 years, double reading of MRI } \\
\text { images, but single reading of } \\
\text { macroscopic examinations; wide range } \\
\text { of sensitivity and specificity }\end{array}$ \\
\hline Bredella (2001) & Radiology & $\begin{array}{l}\text { Consecutive patients; grading of cartilage } \\
\text { lesions considered; exact numbers of TP, } \\
\text { TN, FP, and FN results can be derived }\end{array}$ & $\begin{array}{l}\text { Only } 20 \text { patients; arthroscopers not blinded } \\
\text { to MRI results; MRI interpretation } \\
\text { performed by consensus of two readers, } \\
\text { not independently }\end{array}$ \\
\hline Harman (2003) & $\begin{array}{l}\text { Radiology, orthopaedic } \\
\text { surgery }\end{array}$ & $\begin{array}{l}50 \text { knees of } 42 \text { patients; grading of cartilage } \\
\text { lesions considered; arthroscoper blinded } \\
\text { to MRI results }\end{array}$ & $\begin{array}{l}\text { No confidence intervals for diagnostic } \\
\text { parameters calculated; MRI interpretation } \\
\text { performed by consensus of two readers, not } \\
\text { independently }\end{array}$ \\
\hline Kladny (1995) & $\begin{array}{l}\text { Orthopaedic } \\
\text { rheumatology }\end{array}$ & $\begin{array}{l}\text { Directly comparing } 0.2 \text { - vs. } 1.5-\mathrm{T} \text { MRI and } \\
\text { using arthroscopy as a reference for both; } \\
\text { all patients gave informed consent; wide } \\
\text { age range of patients (14-70 years); short } \\
\text { interval between MR imaging and } \\
\text { arthroscopy; grading of cartilage } \\
\text { lesions considered }\end{array}$ & $\begin{array}{l}\text { Only } 22 \text { patients examined, cartilage defects } \\
\text { detected only in } 6 / 22 \text { patients; no sensitivity } \\
\text { and specificity values for knee cartilage } \\
\text { lesions displayed; arthroscopers not blinded } \\
\text { to MR imaging results }\end{array}$ \\
\hline Kreitner (1999] & $\begin{array}{l}\text { Radiology, orthopaedic } \\
\text { surgery, statistics }\end{array}$ & $\begin{array}{l}75 \text { patients prospectively examined with } \\
\text { MRI and arthroscopy, with } 31 \text { patients } \\
\text { showing cartilage lesions; confidence } \\
\text { intervals for sensitivity and specificity } \\
\text { intervals calculated; reading } \\
\text { independently performed by an } \\
\text { experienced and a less experienced } \\
\text { radiologist }\end{array}$ & $\begin{array}{l}\text { Low number of patients with cartilage defects, } \\
\text { and large confidence intervals for sensitivity } \\
\text { values; arthroscopers not blinded to MRI } \\
\text { results }\end{array}$ \\
\hline Riel (1999) & $\begin{array}{l}\text { Sports orthopaedics, } \\
\text { radiology }\end{array}$ & $\begin{array}{l}244 \text { consecutive patients prospectively } \\
\text { evaluated, } 62 / 244 \text { showed full- } \\
\text { thickness cartilage lesions }\end{array}$ & $\begin{array}{l}\text { No confidence intervals for diagnostic parameters } \\
\text { calculated; only full-thickness cartilage lesions } \\
\text { detected/considered }\end{array}$ \\
\hline
\end{tabular}

The conclusions of both meta-analyses regarding our research question were that there are no differences between low- and high-field MRI in the detection of meniscal or cruciate ligament tears. However, since the authors did not provide detailed data and also included studies using a field strength of $1.0 \mathrm{~T}$ in their analyses, we decided to recalculate their results. For these recalculations, we included all studies for which an MRI field strength of $<1.0 \mathrm{~T}$ or $\geq 1.5 \mathrm{~T}$ was reported. Table 4 contains a summary of these calculations.

Despite expectations that high-field MRI raises the likelihood of detecting those lesions, this was not the case. On the contrary, there were practically no differences in the diagnostic performance of low- versus high-field MRI for the detection of meniscal or cruciate ligament tears detectable (Table 4).

A systematic review in which low- and high-field strength MRI of cartilage defects or osteoarthritis of the knee were compared could not be found in the literature. However, we identified two relevant systematic reviews summarising highfield strength MRI results [10, 11]. Details of these reviews are displayed in Table 5.
The identified studies used a large variety of imaging techniques including sequences, slice size, plane of data collection, positioning of patients, and types of scanners. This and the varying qualification and experience of involved radiologists and arthroscopers may have contributed to the wide range of diagnostic performance results. Altogether, the results show that high-field MRI seems to be more specific than sensitive for the detection or grading of cartilage defects of the knee and that the sensitivity is associated with the (severity) grade of the lesions.

We also extracted data from the six identified studies [13-18] that used low-field strength imaging for the evaluation of cartilage defects in the human knee. The findings were diverse and can be summarised as follows: Using low-field MRI, high-grade lesions are detected, but often low-grade lesions are overlooked. The experience of the radiologist plays an important role in the diagnostic process as does the selection of the appropriate examination parameters. The extensive data extraction table can be obtained from the authors upon request. The results suggest that there is insufficient evidence 
Table 3 Meta-analyses that contained MRI studies with different field strengths

\begin{tabular}{|c|c|c|}
\hline & Oei et al. [2] & Smith et al. [9] \\
\hline No. and content of included articles & $\begin{array}{l}29 \text { MR imaging articles, comprising } 27 \\
\text { studies on both menisci, } 23 \text { studies on } \\
\text { ACL tears, and } 12 \text { studies on PCL tears }\end{array}$ & $\begin{array}{l}53 \text { MR imaging articles, all comprising } \\
\text { studies on complete anterior cruciate } \\
\text { ligament (ACL) tears }\end{array}$ \\
\hline Articles published in the years & $1991-2000$ & $1986-2009$ \\
\hline \multirow[t]{6}{*}{ Inclusion criteria for the articles } & $\geq 30$ patients studied & No cadaveric and animal studies \\
\hline & Arthroscopy used as a reference standard & $\begin{array}{l}\text { Arthroscopy or open surgery findings } \\
\text { used as a reference standard }\end{array}$ \\
\hline & Magnetic field strength reported & No restrictions \\
\hline & Positivity criteria for MR imaging defined & No arthrogram studies \\
\hline & $\begin{array}{l}\text { Absolute numbers of true-positive, false- } \\
\text { positive, true-negative, and false- } \\
\text { negative results available or derivable }\end{array}$ & $\begin{array}{l}\text { Absolute numbers of true-positive, } \\
\text { false-positive, true-negative, and false- } \\
\text { negative results available or derivable }\end{array}$ \\
\hline & Only English-language articles & No restrictions \\
\hline $\begin{array}{l}\text { Results with regard to differences } \\
\text { in diagnostic performance } \\
\text { between low- and high-field } \\
\text { MR imaging }\end{array}$ & $\begin{array}{l}\text { The results of the separate pooled } \\
\text { weighted analyses for various } \\
\text { categories of magnetic field strengths } \\
\text { (not tabulated) suggested a modest } \\
\text { trend toward better diagnostic } \\
\text { performance for higher field strength } \\
\text { categories. None of the differences } \\
\text { were found to approach statistical } \\
\text { significance, however, and the } \\
\text { confidence intervals were all wide }\end{array}$ & $\begin{array}{l}\text { No differences between low- and high- } \\
\text { field MR imaging in the detection of } \\
\text { ACL tears }\end{array}$ \\
\hline
\end{tabular}

for low-field MRI because only a few studies with small samples and equivocal findings have been published.

\section{Discussion}

Several review articles on low-field versus high-field MRI of the extremities agree that low-field strength MRI systems provide the ability to diagnose substantial pathologies within the ligaments and menisci of the knee $[3,9,19]$. This is in accordance with our findings. All pooled sensitivity values were high, except for those values that were observed for the lateral meniscus. But this was not associated with the used MRI field strength. A reason for the latter result may be that radiologists are undersensitised for recognising lateral meniscal lesions because they are less common than medial meniscal or ACL tears [19].

However, it should be stressed that specialised training is required for quality control and image interpretation in lowfield MRI. According to Ghazinoor et al., a reason for insufficient diagnostic accuracy of low-field MRI systems is the use of large FOVs and poor-in-plane resolution [19]. They also recommend the performance of a STIR sequence to further improve the system's diagnostic capabilities. The importance of the radiologist's qualification was shown in an Austrian study [20]: In MRI examinations of knee pathologies, the

Table 4 Pooled sensitivity and specificity values of MR imaging for the detection of various knee injuries, including $95 \%$ confidence intervals and separated by field strength

\begin{tabular}{lllrrrr}
\hline & \multicolumn{1}{c}{ Field strength of $<1.0 \mathrm{~T}$} & \multicolumn{3}{c}{ Field strength of $\geq 1.5 \mathrm{~T}$} \\
\hline Type of injury & Sensitivity & Specificity & $\mathrm{n}$ & Sensitivity & Specificity \\
Medial meniscal tear & $0.91[0.88 ; 0.93]$ & $0.87[0.85 ; 0.90]$ & 1138 & $0.94[0.93 ; 0.95]$ & $0.87[0.85 ; 0.89]$ & 2173 \\
Lateral meniscal tear & $0.76[0.70 ; 0.81]$ & $0.95[0.93 ; 0.96]$ & 1155 & $0.78[0.75 ; 0.82]$ & $0.95[0.94 ; 0.96]$ & 2206 \\
PCL complete tear & $1.00[0.80 ; 1.00]$ & $0.98[0.96 ; 0.99]$ & 555 & $0.88[0.62 ; 0.98]$ & $0.98[0.97 ; 0.99]$ & 961 \\
ACL complete tear & $0.92[0.87 ; 0.95]$ & $0.93[0.90 ; 0.94]$ & 912 & $0.94[0.91 ; 0.96]$ & $0.95[0.93 ; 0.96]$ & 1514 \\
ACL complete tear* & $0.92[0.89 ; 0.95]$ & $0.93[0.91 ; 0.95]$ & 1109 & $0.92[0.90 ; 0.94]$ & $0.95[0.94 ; 0.96]$ & 3112 \\
\hline
\end{tabular}

$n=$ number of cases; * results from the studies included in Smith et al. [9], all other results refer to the studies included in the meta-analysis of Oei et al. [2] Forest plots can be obtained from the corresponding author upon request 
Table 5 Description of the methodology and results of the two systematic reviews on the diagnostic performance of high-field MRI of cartilage defects and knee osteoarthritis

\begin{tabular}{|c|c|c|}
\hline & Quatman et al. [11] & Harris et al. [10] \\
\hline No. and content of included articles & $\begin{array}{l}27 \text { MRI articles, comprising } 27 \text { studies } \\
\text { on articular cartilage abnormalities, } \\
\text { and } 20 \text { studies on knee osteoarthritis }\end{array}$ & $13 \mathrm{MRI}$ articles on articular cartilage defects \\
\hline Articles published in the years & 1988-2010 & 1994-2009 \\
\hline \multirow[t]{4}{*}{ Inclusion criteria for the articles } & Human knee & Human knee \\
\hline & Arthroscopy used as a reference standard & Arthroscopy used as a reference standard \\
\hline & Magnetic field strength of $\geq 1.5 \mathrm{~T}$ & Magnetic field strength of $\geq 1.5 \mathrm{~T}$ \\
\hline & MRT sequences reported & No MRT outcomes after cartilage surgery \\
\hline \multirow{8}{*}{$\begin{array}{l}\text { Sensitivity of MRI in articular cartilage } \\
\text { defects in the various locations }\end{array}$} & Medial tibial plateau: $0.17-0.96$ & \\
\hline & Lateral tibial plateau: $0.00-0.58$ & \\
\hline & Medial femoral condyle: $0.28-1.00$ & \\
\hline & Lateral femoral condyle: $0.33-1.00$ & \\
\hline & Trochlea: $0.55-1.00$ & Trochlea: $0.62-1.00$ \\
\hline & Patella: $0.21-1.00$ & Patella: $0.00-0.95$ \\
\hline & Patellofemoral compartment: $0.44-0.95$ & \\
\hline & Tibiofemoral compartment: $0.42-0.81$ & \\
\hline $\begin{array}{l}\text { Specificity of MRI in articular } \\
\text { cartilage defects }\end{array}$ & $\begin{array}{l}>0.85 \text { in almost all studies and locations, } \\
\text { with the exception of two studies (patella: } \\
0.75 \text {; lateral femoral condyle: } 0.78 \text { ) }\end{array}$ & Patella: $0.62-1.00$. trochlea: $0.81-0.97$ \\
\hline Sensitivity of MRI in early osteoarthritis & $0.00-0.86$ & \\
\hline Specificity of MRI in early osteoarthritis & $0.48-0.95$ & \\
\hline Sensitivity of MRI in advanced osteoarthritis & $0.47-0.98$ & \\
\hline Specificity of MRI in advanced osteoarthritis & $0.60-1.00$ & \\
\hline
\end{tabular}

number of false reports was dependent on the radiologist rather than on the MRI field strength, and the rate of false interpretations was significantly higher in the less experienced group of radiologists.

Relying on low-field imaging for the detection of meniscal and cruciate ligament injuries may, however, lead to a specific problem in case of equivocal findings: Since most radiologists' level of confidence is superior with high-field MRI, such findings after low-field MRI may increasingly lead to second examinations on high-field units. Another problem with the use of low-field MR systems is the increasing selfreferral rates, e.g. by non-radiologists who install these systems in their offices. It was shown that self-referring physicians used imaging procedures more frequently than those referring to a radiologist, resulting in an increase of imaging charges for self-referring physicians [6].

In contradistinction, accurate diagnosis, and characterisation of articular cartilage lesions are difficult, if not impossible, in the

Table 6 Take-home table

Conclusions from results displayed in Tables 4 and 5

Diagnostic performance of low- and high-field MRI in meniscus and cruciate ligament injuries is equal

Diagnostic performance of low-field MRI in knee cartilage defects and osteoarthritis is acceptable

Diagnostic performance of low-field MRI in knee cartilage defects and osteoarthritis is good

Recommendations for use and renumeration of low-field MRI in meniscus and cruciate ligament injuries

MRI on extremity scanners is best performed by musculoskeletal-trained radiologists with experience in reading images obtained on low-field systems, working closely with the referring clinician

MRI should not replace clinical diagnosis, but be used in connection with clinical findings and history to provide a more complete picture, especially with complex injuries

Since the level of confidence in decision-making is reported significantly superior with high-field imaging, equivocal findings with low-field units may lead to an increased number of second examinations on high-field units

The rates of self-referral, e.g. by non-radiologists who install these systems in their offices, should be controlled 
setting of low-field strength imaging $[3,6,19]$. All in all, the increased noise inherent in low-field MRI seems to affect the detection of cartilage abnormalities when compared to high-field imaging. Full-thickness cartilage defects are easier to appreciate than lower-grade chondral abnormalities.

However, also in high-field strength MRI the results, specifically with regard to sensitivity, are unsatisfactory and often below the usual clinical standard. Recently, Strickland and Kijowski presented a review article in which they discuss the current state of MR cartilage imaging and the existing problems [21]. There is a large variety of MRI techniques available for evaluating articular cartilage. Limited spatial resolution, supoptimal tissue contrast, and artefacts remain major hurdles in the development of clinically useful sequences. The authors believe that the main factor that limits the ability of currently available MRI techniques to identify early cartilage degeneration is suboptimal spatial resolution. Thus, it is expected that MRI systems with higher field strengths (e.g., 3.0 T) will improve cartilage imaging as images are produced with higher spatial resolution and decreased slice thickness without reducing the signal-to-noise ratio or prolonging acquisition time.

Some authors say that the MRI techniques recommended in the literature at present are not able to replace arthroscopy for diagnosis of cartilage damage in the knee [22]. However, according to the results of a randomised controlled trial the use of MRI in patients with chronic knee problems, in whom surgery was being considered, did not increase costs overall, was not associated with worse outcomes, and avoided surgery in a significant proportion of patients [1].

Our review showed that the diagnostic reliability and utility of low-field MRI in detecting meniscus and cruciate ligament tears are comparable to those obtained from conventional units operating at higher magnetic fields (see also Table 6). This is supported by newer primary studies not included in this review, e.g. [23]. The accurate diagnosis and characterisation of cartilage lesions, however, are difficult, if not impossible, in the setting of low-field strength imaging.

Therefore, we can recommend the use and remuneration of low-field strength systems in suspicious meniscus and cruciate ligament injuries with the constraints or specific considerations displayed in Table 6.

Based on the existing evidence, we cannot recommend the use of low-field-strength systems for the diagnosis and grading of knee cartilage defects and osteoarthritis. Since the sensitivity of high-field MRI for chondral lesions is also suboptimal, we recommend referring those patients to specialised centers or well-trained individuals using up-to-date equipment, respectively.

Funding This work was funded by the Main Association of Austrian Social Insurance Institutions.
Conflicts of interests None

\section{References}

1. Bryan S, Bungay HP, Weatherburn G, Field S. Magnetic resonance imaging for investigation of the knee joint: a clinical and economic evaluation. Int J Technol Assess Health Care. 2004;20:222-9.

2. Oei EHG, Nikken JJ, Verstijnen ACM, Ginai AZ, Hunink MG. MR imaging of the menisci and cruciate ligaments: a systematic review. Radiology. 2003;226:817-48.

3. Tavernier T, Cotten A. High- versus low-field MR imaging. Radiol Clin N Am. 2005;43:673-81.

4. Masciocchi C, Barile A, Satragno L. Musculoskeletal MRI: dedicated systems. Eur Radiol. 2000;10:250-5.

5. Nikken JJ, Oei EHG, Ginai AZ, et al. Acute peripheral joint injury: cost and effectiveness of low-field-strength MR imaging - results of randomized controlled trial. Radiology. 2005;236:958-67.

6. Sanal HT, Cardoso F, Chen L, Chung C. Office-based versus highfield strength MRI. diagnostic and technical considerations. Sports Med Arthrosc Rev. 2009;17:31-9.

7. Puig S, Felder-Puig R. Evidenzbasierte Radiologie: Ein neuer Ansatz zur Bewertung von klinisch angewandter radiologischer Diagnostik und Therapie. Fortschr Roentgenstr. 2006;178:671-9.

8. The Evidence-Based Radiology Working Group. Evidence-based radiology: a new approach to the practice of radiology. Radiology. 2006;220:586-75.

9. Smith TO, Lewis M, Song F, Toms AP, Donell ST, Hing CB. The diagnostic accuracy of anterior cruciate ligament rupture using magnetic resonance imaging: a meta-analysis. Eur J Orthop Traumatol. 2012;22:315-26.

10. Harris JD, Brophy RH, Jia G, et al. Sensitivity of magnetic resonance imaging for detection of patellofemoral articular cartilage defects. Arthroscopy. 2012;11:1728-37.

11. Quatman CE, Hettrich CM, Schmitt LC, Spindler KP. The clinical utility and diagnostic performance of magnetic resonance imaging for identification of early and advanced knee osteoarthritis. Am J Sports Med. 2011;39:1557-67.

12. Whiting P, Rutjes AWS, Reitsma JB, Bossuyt PMM, Kleijnen J. The development of QUADAS: a tool for the quality assessment of studies of diagnostic accuracy included in systematic reviews. BMC Med Res Methodol. 2003;3:25.

13. Ahn JM, Kwak SM, Kang HS, et al. Evaluation of patellar cartilage in cadavers with a low-field-strength extremity-only magnet: comparison of MR imaging sequences, with macroscopic findings as the standard. Radiology. 1998;208:57-62.

14. Bredella MA, Losasso C, Moelleken SC, Huegli RW, Genant HK, Tirman PF. Three-point Dixon chemical-shift imaging for evaluating articular cartilage defects in the knee joint on a low-fieldstrength open magnet. AJR. 2001;177:1371-5.

15. Harman M, Ipeksoy U, Dogan A, Arslan H, Etlik O. MR arthrography in chondromalacia patellae diagnosis on a low-field open magnet system. J Clin Imag. 2003;27:194-9.

16. Kladny B, Glückert K, Swoboda B, Beyer W, Weseloh G. Comparison of low-field ( 0.2 Tesla) and high-field (1.5 Tesla) magnetic resonance imaging of the knee joint. Arch Orthop Trauma Surg. 1995;114:281-6.

17. Kreitner K-F, Hansen M, Schadmand-Fischer S, Krummenauer F, Runkel M. Niederfeld-MR-Tomographie des Kniegelenkes: ergebnisse einer prospektiven, arthroskopisch kontrollierten Studie. Fortschr Roentgenstr. 1999;170:35-40.

18. Riel K-A, Reinisch M, Kersting-Sommerhoff HN, Merl T. 0.2Tesla magnetic resonance imaging of internal lesions of the knee 
joint: a prospective arthroscopically controlled clinical study. Knee Surg Sports Traumatol Athrosc. 1999;7:37-41.

19. Ghazinoor S, Crues JV, Crowley C. Low-field musculoskeletal MRI. J Magn Res Imag. 2007;25:234-44.

20. Krampla W, Rosel M, Svoboda K, Nachbagauer A, Gschwantler M, Hruby W. MRI of the knee: how do field strength and radiologist's experience influence diagnostic accuracy and interobserver correlation in assessing chondral and meniscal lesions and the integrity of the anterior cruciate ligament? Eur Radiol. 2009;19:1519-28.
21. Strickland CD, Kijowski R. Morphologic imaging of articular cartilage. Magn Reson Imaging Clin N Am. 2011;19: 229-48.

22. Crawford R, Walley G, Bridgman S, Maffulli N. Magnetic resonance imaging versus arthroscopy in the diagnosis of knee pathology, concentrating on meniscal lesions and ACL tears: a systematic review. Br Med Bull. 2007;84:5-23.

23. Chen HN, Dong QR, Wang Y. Accuracy of low-field MRI on meniscal tears. GRM. 2014;13:4267-71. 